\title{
La enseñanza interdisciplinaria de las Relaciones Públicas en las universidades de España y México. Un estudio exploratorio
}

\author{
Recibido: 13 de septiembre de 2010 \\ Aceptado: 19 de abril de 2011 \\ Publicado: 31 de octubre de 2011
}

\author{
Marco Tulio Flores Mayorga \\ marcotulio.flores@uab.cat \\ David Roca Correa \\ david.roa@uab.es \\ (Universidad Autónoma de Barcelona - España)
}

Resumen: En el presente artículo se analiza la situación actual de los modelos educativos de las Relaciones Públicas de España y México. Además, se identifican las asignaturas de los planes de estudio diseñados para la didáctica de las RR. PP. Se utiliza una muestra exploratoria de las 11 universidades con mayor tradición en estudios de Relaciones Públicas y Comunicación en España y México. La metodología del estudio se basa en la técnica de análisis de contenido del currículo formativo de los planes de estudio del grado de RR. PP. Los resultados revelan que las Relaciones Públicas en las universidades españolas ganan presencia (20\%), mientras que en México no es tan fuerte (14\%) y se sitúan por detrás de los cursos de Mercadotecnia, Economía, Administración y Negocios. Dentro de las asignaturas interdisciplinarias, las ciencias sociales representan un $10 \%$ en las universidades españolas y un $14.8 \%$ en las mexicanas.

Palabras clave: Relaciones Públicas, currículo formativo, interdisciplinar, Espacio Europeo de Educación Superior, México, España.

Abstract: This research analyzes the current educational models of Public Relations in Spain and Mexico. It also identifies the subjects of the curriculum designed for the teaching of Public Relations. It uses an exploratory sample of 11 universities with long experience and tradition studies of public relations and communications in Spain and Mexico. The study methodology is based on the content analysis of the training curriculum of undergraduate public relations. The results reveal that public relations in Spanish universities acquire presence (20\%). On the other hand, its presence in Mexico is not as strong (14\%) and lag behind marketing, economics, business and administration. The 
interdisciplinary courses represent $80 \%$ in Spain and $86 \%$ in Mexico. Among these, the presence varies: the social sciences make up 10\% in Spanish universities and $14.8 \%$ in Mexico, philosophy and literature are the presence of $2 \%$ and $0.4 \%$ respectively.

Key words: Public Relations, Syllabus, Interdisciplinary, European Higher Education, Mexico, Spain.

\section{Introducción}

Las Relaciones Públicas cuentan con un cuerpo teórico que puede alcanzar una total madurez (Grunig \& Hunt, 2003), aunque se continuó el debate por su indefinición como ciencia (Arceo, 2004; Magallón, 2004) y profesión (L'Etang, 2009), así como el desconocimiento de su función en el ámbito organizacional (Sriramesh \& Hornaman, 2006; Arceo, 2004). Esta incertidumbre condiciona su legitimación en la enseñanza superior, en donde las Relaciones Públicas están siendo reemplazadas por otras denominaciones como pueden ser "Comunicación Corporativa", "Comunicación Institucional”, "Comunicación Organizacional" y el perfil, de reciente surgimiento, Community Manager. Esta sustitución se debe a la mala reputación generada desde sus orígenes, así como a los intereses académicos y al entorno económico, político y social. Por estas razones, el presente estudio exploratorio pretende: a) analizar la situación actual de los modelos educativos de las relaciones públicas entre España y México; y b) identificar la orientación predominante de las asignaturas interdisciplinarias en cada una de las universidades que imparten estudios de Relaciones Públicas. Es una investigación que mira al interior del sistema educativo superior de España y México.

\subsection{El antecedente norteamericano en la enseñanza de las Relaciones Públicas}

Las RR. PP. ingresaron en los planes de estudio de las universidades norteamericanas a través de las escuelas de Periodismo, debido al dominio del modelo de información pública ${ }^{1}$ y de la necesidad de las técnicas periodísticas para los profesionales de que seguían ese modelo (Grunig \& Hunt, 1984).

Desde que Edward L. Bernays impartió en la Universidad de New York el primer curso de Relaciones Públicas en 1923 ("Public Relations. A course on theory and practical method") aparecieron un sinnúmero de materias similares. Ese suceso histórico permitió que algunos de los practicantes de las Relaciones Públicas conectasen, a partir de entonces, con unas coordenadas académicas y de gestión empresarial e institucional (Arceo, 2004).

En 1947 se esbozó el primer programa de maestría en Relaciones Públicas, sirviendo de referencia años después cuando muchas instituciones empezaron a ofertar programas en esa especialidad, como el caso del Dorset Institute, primer organismo en el Reino Unido en ofrecer la licenciatura en 1989 (Pratt \& Ogbondah, 1996).

1.- En el modelo de información pública, el público debe ser informado de forma unidireccional. Aparece a partir de 1920. 


\subsection{El desarrollo de los estudios de las Relaciones Públicas en España}

Es necesario destacar la incidencia del periodismo. En la década del treinta, dicha especialidad tuvo escuelas privadas auspiciadas por la Iglesia católica. Un ejemplo vigente de ello ha sido la Fundación Universitaria San Pablo CEU, creada en 1933 por la Asociación Católica de Propagandistas (ACdP).

Durante los últimos años del franquismo, los Centros de Nuevas Profesiones (de naturaleza privada, pero dirigidos por personas con vinculaciones al Ministerio de Información y Turismo de entonces) se ocuparon de los estudios de Turismo, Publicidad y, en menor medida, de las Relaciones Públicas. El principal criterio era el de las matrículas o demanda (Noguero, A, 2011). En el ámbito docente, Juan Beneyto impartió un seminario denominado "Los medios de la información contemporánea" (1954), en el que analiza algunos aspectos de la actividad de las RR. PP. (Noguero, 2004).

Las Relaciones Públicas como asignatura se empieza a ofertar en España dentro del Plan de Estudios de Periodismo (1967), impartido por la Escuela Oficial de Periodismo, bajo el rótulo "Fundamentos de Psicología y Teoría y Técnica de la Propaganda y de las Relaciones Públicas" (Orden ministerial 20/04/1967, BOE 4 de mayo).

En 1969 es reconocida oficialmente la Escuela Superior de Relaciones Públicas, la primera especializada en la materia, adscrita a la Universidad de Barcelona (BOE de 26.08.69). Dos años después se regulan los estudios de Periodismo y Comunicación Social, creándose las primeras Facultades de Ciencias de la Información. La Universidad Complutense de Madrid inició con "Periodismo", "Ciencias de la imagen visual y auditiva" y "Publicidad"; y en la Autónoma de Barcelona, con "Periodismo" (RD 2478/1971). No fue hasta 1974 cuando se aprobó provisionalmente el plan de estudios de la licenciatura de Publicidad y Relaciones Públicas (Xifra, 2007), ampliándose la licenciatura en Publicidad, creada en 1972 (RD 2140/1972). En la actualidad, los estudios de Comunicación en el sistema educativo español universitario incluyen los grados de Periodismo, Comunicación Audiovisual, Publicidad y Relaciones Públicas.

\subsection{España en el espacio europeo de educación superior}

La enseñanza educativa superior en la Península ha transitado por varios hechos significativos que se acrecentaron cuando se debate el Plan Bolonia, el cual trajo consigo la creación del Espacio Europeo de Educación Superior (EEES), aprobado en 1999 por 29 países. Hasta hoy, los planes de estudios del Grado de Publicidad y Relaciones Públicas de treinta universidades españolas públicas y privadas han sido evaluados favorablemente por la Agencia Nacional de Evaluación de la Calidad y Acreditación (Verifica ANECA 2011)2 Según datos provisionales del Servicio de Estadística Universitaria del Ministerio de Educación, en el período 2007-2010 se matricularon 48485 estudiantes de la licenciatura en Publicidad y Relaciones Públicas; y 3169 estudiantes en el período 2008-2010. El año 2010 fue el plazo fijado para iniciar los grados con la nueva estructura del EEES.

2.- La ANECA contribuye a la mejora de la calidad del sistema de educación superior mediante la evaluación, certificación y acreditación de enseñanzas, profesorado e instituciones, a través de siete programas, entre ellos el Verifica. 
Entre otros objetivos del proceso Bolonia están la adopción de un sistema legible y comparable de titulaciones, la creación de un sistema de créditos europeo, fortalecer la movilidad académica y administrativa, así como centrar la enseñanza en el estudiante.

Un hecho a destacar es que en el 2007 se establece la ordenación de las enseñanzas universitarias oficiales (RD 1393/2007) en la cual se resalta la autonomía universitaria y la flexibilización de la organización de las enseñanzas universitarias.

Los estudios constan de tres ciclos de grado con una duración de cuatro años, cubriendo 240 European Credit Transfer System (ECTS), procurando ofrecer una formación académica y profesional. El número mínimo de horas por crédito es de $25 \mathrm{y}$ el número máximo es de 30 . Existe el suplemento europeo al título universitario oficial ${ }^{3}$ (RD, 1044/2003) que permite reconocer el valor académico y profesional de las titulaciones.

Los grados están diseñados considerando las competencias disciplinares ("saber"), profesionales ("saber hacer") y académicas. Se estructuran en bloques de "formación básica", "obligatoria", "optativas", "prácticas externas" y "trabajo de fin de grado". Los contenidos específicos de los planes de estudio para cada grado son responsabilidad de cada universidad. Los perfiles y salidas profesionales ${ }^{4}$ varían según la universidad. En gran medida se basan en el Libro Blanco ${ }^{5}$ (ANECA, 2005).

Se estima que para el 2015 habrán desaparecido las licenciaturas y estarán activas las primeras generaciones de futuros profesionales que se iniciaron con el Plan Bolonia. La Facultad de Comunicación de la Universidad de Navarra y la Escuela Superior de Relaciones Públicas de la Universidad de Barcelona fueron las primeras en adelantarse en el 2009 con la implementación de su programa de estudios para el Grado de Publicidad y Relaciones Públicas.

\subsection{El desarrollo de los estudios de las Relaciones Públicas en México}

En las universidades mexicanas, los estudios no son homogéneos: predomina la carrera de Ciencias de la Comunicación, donde figuran asignaturas en estudios de la Comunicación, Periodismo, Publicidad y Relaciones Públicas. Otras universidades ofrecen la especialización, ya sea en Comunicación Audiovisual, Periodismo y Comunicación Corporativa. De manera independiente, algunas universidades ofertan la carrera de Comunicación Organizacional, Comunicación Pública, Comunicación Intercultural, Periodismo, Publicidad y Relaciones Públicas.

La Secretaría de Educación Pública registró (en el período 2006-2007) un total de 72224 estudiantes inscritos en programas afines a la comunicación. En el 2009, el número de programas de comunicación en instituciones educativas ascendió a 1006, mientras que la

3.- Es un documento con la información unificada, personalizada para cada título universitario, sobre los estudios cursados, los resultados obtenidos, las capacidades profesionales adquiridas y el nivel de su titulación en el sistema nacional de educación superior.

4.- Los perfiles más comunes son: Dirección de Comunicación, Investigación, Consultor Estratégico en Publicidad y Relaciones Públicas; Investigadores, Planificadores y Compradores de Medios; Creativo y Diseñador; y Gestor de Comunicación Corporativa.

5.- Es un análisis de los estudios de comunicación en España para su adaptación a la convergencia europea. 
Asociación Nacional de Universidades e Instituciones de Educación Superior (ANUIES) reportó 462 en el año 2007 (Felafacs, 2009).

Destacan actualmente 94 programas de estudios de comunicación, los cuales han sido evaluados y reconocidos por los órganos acreditadores: 36 por los Comités Interinstitucionales para la Evaluación de la Educación Superior (CIEES) ${ }^{6}, 26$ por el Consejo para la Acreditación de la Comunicación (CONAC) ${ }^{7}$ y 32 por la Asociación para la Acreditación y Certificación en Ciencias Sociales (ACCECISO) ${ }^{8}$.

Alrededor de 20 instituciones públicas y privadas ofrecen estudios específicos de Relaciones

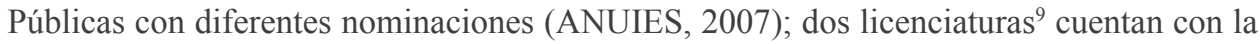
acreditación de los CIEES y las restantes actualizan sus programas, siendo validados por los departamentos o centros de adscripción al que pertenecen.

Las Relaciones Públicas en México surgen en el ámbito profesional con la primera agencia de RR. PP. creada en 1949 por Federico Sánchez Fogarty. Tiempo después, esta especialidad se incorpora a la enseñanza del periodismo y la comunicación. Aunque ya en la presidencia de Lázaro Cárdenas del Río (1930) la función de las Relaciones Públicas era denominada "Prensa y Propaganda Política", cuya tarea principal era la gestión de la información (Long, 2004).

Los estudios de comunicación surgen al fundarse la Escuela de Periodismo Carlos Septién García (1949), la primera en el país. Poco después se abren las carreras de Periodismo en la Universidad Autónoma de México (1951) y en la Universidad Veracruzana (1954).

En 1960, la Universidad Iberoamericana incluyó (por primera vez en América Latina) las Relaciones Públicas como materia opcional en la Licenciatura en Ciencias y Técnicas de la Información.

Durante el período 1973-1974, la Universidad Veracruzana incluyó asignaturas de Relaciones Públicas (Universidad Veracruzana, 2011). Por su parte, la Universidad Latinoamericana abre la licenciatura en Comunicación y Relaciones Públicas (1976), mientras que en 1982 la Universidad del Valle de México creó la primera licenciatura bajo la denominación específica de Relaciones Públicas (González, 2011).

Un hecho trascendental para los estudios de comunicación en México ha sido la creación del modelo educativo de las Universidades Interculturales $(2003)^{10}$. En dichas casas de estudio se ofertan la carrera de Comunicación Intercultural, en la que se imparten las asignaturas de Comunicación, Publicidad, Mercadotecnia y Periodismo.

6.- Evalúan las funciones y programas académicos de universidades públicas y privadas.

7.- Es reconocida por el Consejo para la Acreditación de la Educación Superior (COPAES). Se encarga de acreditar los programas de estudios relacionados con las ciencias de la comunicación.

8.- Realiza procesos de evaluación y de acreditación de los programas educativos en las siguientes disciplinas: Ciencia Política, Sociología, Trabajo Social, Relaciones Internacionales, Antropología, Administración Pública, Comunicación, Geografía, Ciencias de la Información Documental y Bibliotecología.

9.- Mercadotecnia y Relaciones Públicas de la Universidad Autónoma de Guerrero y Publicidad y Relaciones Públicas de la Universidad Veracruzana.

10.- Está dirigido a jóvenes de diversos sectores sociales, interesados en el desarrollo de los pueblos y comunidades indígenas. Existen nueve universidades que conforman la red de Universidades Interculturales de México. 


\subsection{El modelo educativo de las Relaciones Públicas en México}

Los estudios de RR. PP. están presentes en el nivel técnico superior universitario, profesional asociado y licenciatura, así como en el ámbito de posgrado, en especialidades y maestrías.

Los planes de estudio de licenciatura están estructurados por competencias de formación básica, disciplinaria, optativas, de elección libre, servicio social ${ }^{11}$, prácticas profesionales y seminarios de investigación.

El modelo educativo que todavía prevalece desde la década del sesenta es el "modelo polivalente", por medio del cual se pretendió trascender la autonomía relativa de profesiones y diferentes disciplinas (tales como Publicidad, Relaciones Públicas, Periodismo) subordinándolas a la perspectiva integradora de las Ciencias de la Comunicación (Islas, 2004). Dicho modelo fue introducido por el Centro Internacional de Estudios Superiores de Comunicación para América Latina (CIESPAL), en un seminario de Río de Janeiro (1965) cuando el centro intentaba promover la reorientación de las carreras de periodismo en los Institutos de comunicación o institutos de ciencias de comunicación colectiva (Molleda, 2001).

El valor de una licenciatura es de 300 créditos como mínimo y 450 como máximo. Cada cuerpo colegiado se encarga de establecer el número exacto, siempre dentro de los límites señalados (Acuerdo Nayarit, 1972).

Los estudiantes adquieren conocimiento y habilidades en el diseño de estrategias de Comunicación Audiovisual, Creatividad, Propaganda, Imagen, Publicidad, Relaciones Públicas y Mercadotecnia, así como en investigación y docencia en Publicidad y Relaciones Públicas.

\section{Estado de la cuestión}

La investigación introspectiva (Pavlik, 1987) comprende los cambios constantes del entorno en el que se desarrollan las Relaciones Públicas. En el ámbito educativo se han realizado estudios sobre el currículo formativo de las RR. PP. en el nivel superior (Matilla, Hernández y Losada, 2010; Observatori de la Càtedra Dircom.Cat, 2010; Xifra, 2007), educación y profesión (Flores, Roca y Tena, 2010; Marques, 2009; Sriramesh y Hornaman, 2006), maestría (Fuentes, 2010) y doctorado (Castillo y Xifra, 2006).

El estudio realizado por Xifra (2007), sobre la enseñanza de las Relaciones Públicas, revela que las asignaturas referidas a la teoría, orígenes, principios y a la práctica profesional de las RR. PP. están presente en las 32 universidades que imparten la licenciatura de Publicidad y Relaciones Públicas; la materia de Planeación y Gestión de las Relaciones Públicas en 24 (75\%); Escritura y Producción de las RR. PP. en 19 (59.37\%); Investigación y Evaluación de las RR. PP. en 16 (50\%). Con menor presencia, Derecho y Ética de las Relaciones Públicas en 9 universidades (28.12\%); Práctica y Aplicación de las RR. PP. en

11.- Se inicia durante la presidencia del general Lázaro Cárdenas en 1936, año en el que fue creado el Instituto Politécnico Nacional, para dar respuesta a las necesidades técnicas. En 1952 se hizo una obligación para todos los estudiantes de enseñanza superior en México. 
5 (15.62\%); y la experiencia de trabajo supervisado en Relaciones Públicas en 4 (12.5\%). De las asignaturas interdisciplinarias, Mercadotecnia y Comunicación Oral y Escrita se enseñan en todas las universidades; Comportamiento del Consumidor en 29 (90.62\%); Psicología Social en 27 (84.37\%); Sociología en 25 (78.12\%) y Economía en 22 (70.4\%).

Otro estudio a nivel universitario en España (Matilla, Hernández y Losada, 2010) muestra que las asignaturas de Publicidad (61.34\%) tienen una mayor presencia que las de RR. PP. $(19.12 \%)$ en la carrera. En cierta medida, en las universidades catalanas estos datos varían pues las materias publicitarias alcanzan un $53.21 \%$ frente al $22.02 \%$ de las referidas a las Relaciones Públicas (DirCom.Cat, 2010).

Respecto a la investigación teórica sobre Relaciones Públicas, un análisis bibliográfico de 65 tesis doctorales (Xifra y Castillo, 2006), revela el predominio de la investigación aplicada con 47 tesis $(72.31 \%)$, en detrimento de 12 codificadas como introspectivas $(18.46 \%)$ y 2 de elaboración teórica (3.08\%). Dentro de la línea introspectiva, existen dos tesis que abordan las Relaciones Públicas desde la pedagogía y educación.

A nivel de maestría en México, un análisis comparativo de cinco universidades mostró la escasa presencia de tesis sobre Relaciones Públicas (Fuentes, 2010). De 356 tesis solo un $3.37 \%$ correspondían a las RR. PP.: una tesis del Instituto Tecnológico de Estudios Superiores de Occidente $(0.28 \%) ; 8$ de la Universidad Iberoamericana (2.24\%); y 3 del Instituto Tecnológico de Estudios Superiores de Monterrey (0.84\%). La Universidad Autónoma de México y la Universidad de Guadalajara no cobijaron tesis sobre esta especialidad.

En otro contexto, Flores, Roca y Tena (2010) descubrieron la incongruencia entre las necesidades empresariales y la enseñanza superior de las Relaciones Públicas en Cataluña (España). En cambio, en los Estados Unidos de América se produce un mayor consenso entre profesores y profesionales. De acuerdo con el estudio de Sriramesh y Hornaman (2006) se subraya que a) un $94 \%$ de los profesores, $90 \%$ de profesionales y $92 \%$ docentesprofesionales, consideran importante los conceptos y estrategias de comunicación y persuasión; b) el 94\% de profesores y el $80 \%$ de docentes-profesionales señalan como relevante los contenidos referidos a la multiculturalidad y problemas mundiales, mientras que solo un $67 \%$ de profesionales están de acuerdo; c) el 52\% de los profesionales, el $83 \%$ de profesores y el 77\% de docentes-profesionales destacan como necesaria la participación en la comunidad; $d$ ) destaca la aplicación de estudios culturales y de género en las Relaciones Públicas. Los profesores y profesionales no consideran relevante la historia de las RR. PP. y de la comunicación organizacional.

En Portugal, un 79\% de profesores y un 53\% de profesionales consideran muy importante las políticas de comunicación y estrategias del lobby en la enseñanza de las Relaciones Públicas. Se puede afirmar que en dicho país existe una fuerte presencia de la Comunicación y una insuficiente representatividad de las RR. PP. (Marques, 2009).

\section{Metodología}


La metodología utilizada para la presente investigación exploratoria ha sido la técnica de análisis de contenido para determinar posibles tendencias, coincidencias, similitudes y diferencias entre los contenidos del currículo formativo de Relaciones Públicas de las universidades españolas y mexicanas. Se analizaron 11 currículos, 6 corresponden al grado español de Publicidad y Relaciones Públicas; y 5 a las licenciaturas de Relaciones Públicas mexicanas (Apéndice A). La información se obtuvo de las páginas electrónicas universitarias entre diciembre de 2010 y junio de 2011.

\subsection{Objetivos}

El estudio exploratorio plantea dos objetivos: a) analizar la situación actual de los modelos educativos de las Relaciones Públicas entre España y México; y b) identificar la orientación predominante de las asignaturas interdisciplinarias en cada una de las universidades que imparten estudios de RR. PP.

\subsection{Problemas de investigación}

$\mathrm{P}_{1}$ : ¿Qué asignaturas de Relaciones Públicas y de otras disciplinas se consideran en los planes de estudio para la enseñanza de las RR. PP. en las universidades españolas y mexicanas?

$\mathrm{P}_{2}:$ ¿Cuál es la orientación predominante de las asignaturas interdisciplinarias en cada una de las universidades que imparten estudios de Relaciones Públicas?

\subsection{Selección de la muestra}

La muestra se seleccionó considerando dos variables: las universidades con mayor tradición en España en la enseñanza de las Relaciones Públicas y que hubieran aparecido en las cinco primeras posiciones en el ranking de carreras del periódico El Mundo ${ }^{12}$ (2009 y 2010): Universidad Autónoma de Barcelona, Universidad Complutense de Madrid, Universidad Jaume I, Universidad de Navarra y Universidad Ramón Llull. Además, se añadió la Universidad de Barcelona, por contar con la primera Escuela Superior de Relaciones Públicas en Europa (1969).

De las universidades mexicanas se eligieron las tres únicas instituciones públicas que ofrecen los estudios de RR. PP.: la Autónoma del Estado de Morelos, la Autónoma de Guerrero y la Veracruzana. Los programas de estudios de la Universidad Veracruzana (2008) y de la Autónoma de Guerrero (2009) cuentan con el reconocimiento de los Comités Interinstitucionales para la Evaluación de la Educación Superior (CIEES). Se incluyó la Universidad Latinoamericana y la Universidad del Valle de México, ambas privadas. La primera por ser una de las pioneras en programar la carrera en Comunicación y Relaciones Públicas (1976) y la segunda por ofertar con el nombre específico de Relaciones Públicas la primera licenciatura (1982), así como por su cobertura en cinco ciudades de México.

\subsection{Procedimiento}

12.- Este ranking se construye a partir de la valoración de los docentes universitarios de una misma carrera. 
Después de haber seleccionado la muestra, se revisaron los planes de estudios de Relaciones Públicas de las universidades mencionadas. La unidad de análisis estuvo compuesta por las asignaturas. Se identificaron un total de 628326 del currículo español (52\%) y 302 del mexicano (48\%). En la Universidad Veracruzana (México) no se pudo acceder a los nombres de las asignaturas optativas pues no estaban disponibles.

El siguiente paso fue codificar las variables externas de los planes de estudio, considerando: a) la rama de conocimiento a la que pertenecen las Relaciones Públicas en las universidades españolas y mexicanas; b) la facultad o centro de adscripción; c) el nombre de los estudios; d) el año de creación o implementación; y e) la duración de los estudios. Respecto a las variables internas de los planes de estudio se codificaron las asignaturas y los números de créditos asignados.

En el análisis realizado por Xifra (2007) se plantean categorías sobre los contenidos de la enseñanza de las RR. PP. en España, clasificándolos en dos tipos de asignaturas: "Interdisciplinarias" y "Relaciones Públicas". Debido a los objetivos de la investigación, se consideraron las dos y se profundizó en la categoría "Interdisciplinarias" para identificar las disciplinas que forman parte de la didáctica de las RR. PP. y para determinar hacía dónde se orientan en base al número de créditos ofertados por cada institución. A dicha categoría se le añadieron seis subcategorías (disciplinas): a) estudios de comunicación (Comunicación, Periodismo, Publicidad y Creatividad); b) Ciencias Sociales y Humanidades (Filosofía y Letras); c) Arte; d) Marketing, Economía, Administración y Negocios; e) tecnologías de la información y comunicación; yff) “otros", donde se etiquetaron las asignaturas relacionadas con competencias generales y académicas, así como el total de créditos de las optativas sin especificar de la Universidad Veracruzana.

Una vez determinada las categorías y subcategorías, se clasificaron las 628 asignaturas dentro de estas. Se pidió a un segundo codificador que agrupara el $20 \%$ de las asignaturas iniciales en las dos categorías de análisis ("Relaciones Públicas" e "Interdisciplinares") y en las 6 subcategorías de las "Interdisciplinares". La confiabilidad entre codificadores (Intercorder Reability) resultó ser de un 95\%.

\section{Resultados}

\subsection{El currículo español}

Xifra (2007) analiza la situación de la enseñanza de las Relaciones Públicas en el nivel superior en España antes de iniciarse con los estudios de grado. La media de las asignaturas de RR. PP. componían un 20\% frente al $80 \%$ de las disciplinas que contribuyen a su enseñanza (tabla 1). En la Universidad Autónoma de Barcelona y en la Universidad Complutense de Madrid destaca la Publicidad, con un 34\% y 33\% respectivamente, frente a las Relaciones Públicas con un 31\% y 18\%. Este predominio de la Publicidad coincide con el resto del currículo formativo español con un $61.34 \%$, mientras que las RR. PP. con un $19.12 \%$ (Matilla, Hernández y Losada, 2010). Similar comportamiento se observa en los planes de estudio de grado de Publicidad y Relaciones Públicas de las universidades 
públicas y privadas de Cataluña, donde la Publicidad sobresale con un 53.21\%, frente a las RR. PP., con sólo un 22.02\% (DirComCat, 2010).

Tabla 1: Clasificación de asignaturas de Relaciones Públicas

e interdisciplinarias en universidades españolas y mexicanas

\begin{tabular}{|c|c|c|c|c|c|c|c|c|c|c|c|c|c|}
\hline Categorías & UAB & UB & UCM & UJ & UNAV & URLL & ESPAÑ̃ & UAEM & UAG & UVM & UV & $\mathbf{U L}$ & MEXICO \\
\hline Relaciones Públicas & $31 \%$ & $28 \%$ & $18 \%$ & $14 \%$ & $7 \%$ & $22 \%$ & $20 \%$ & $8 \%$ & $17 \%$ & $25 \%$ & $9 \%$ & $10 \%$ & $14 \%$ \\
\hline $\begin{array}{l}\text { Interdisciplinarias } \\
\text { (totales) }\end{array}$ & $69 \%$ & $72 \%$ & $82 \%$ & $86 \%$ & $93 \%$ & $78 \%$ & $80 \%$ & $92 \%$ & $83 \%$ & $75 \%$ & $91 \%$ & $90 \%$ & $86 \%$ \\
\hline $\begin{array}{l}\text { Estudios de comunicación } \\
\text { (suma } 1,2 \text { y } 3 \text { ) }\end{array}$ & $52 \%$ & $37 \%$ & $48 \%$ & $57 \%$ & $53 \%$ & $54 \%$ & $50 \%$ & $13 \%$ & $11 \%$ & $18 \%$ & $29 \%$ & $74 \%$ & $29 \%$ \\
\hline $\begin{array}{l}\text { 1. Comunicación } \\
\text { (incluye lenguas) }\end{array}$ & $18 \%$ & $15 \%$ & $15 \%$ & $37 \%$ & $38 \%$ & $31 \%$ & $26 \%$ & $13 \%$ & $9 \%$ & $13 \%$ & $18 \%$ & $64 \%$ & $23 \%$ \\
\hline $\begin{array}{l}\text { 2. Publicidad } \\
\text { (incluye creatividad) }\end{array}$ & $34 \%$ & $19 \%$ & $33 \%$ & $18 \%$ & $11 \%$ & $18 \%$ & $22 \%$ & $0 \%$ & $2 \%$ & $5 \%$ & $11 \%$ & $6 \%$ & $4.8 \%$ \\
\hline 3. Periodismo & $0 \%$ & $3 \%$ & $0 \%$ & $2 \%$ & $4 \%$ & $5 \%$ & $2 \%$ & $0 \%$ & $0 \%$ & $0 \%$ & $0 \%$ & $4 \%$ & $1 \%$ \\
\hline $\begin{array}{l}\text { Ciencias Sociales } \\
\text { y Humanidades }\end{array}$ & $2 \%$ & $10 \%$ & $15 \%$ & $11 \%$ & $13 \%$ & $10 \%$ & $10 \%$ & $26 \%$ & $17 \%$ & $16 \%$ & $6 \%$ & $8 \%$ & $14.8 \%$ \\
\hline Filosofía y letras & $0 \%$ & $3 \%$ & $3 \%$ & $2 \%$ & $5 \%$ & $1 \%$ & $2 \%$ & $0 \%$ & $0 \%$ & $0 \%$ & $2 \%$ & $0 \%$ & $0.4 \%$ \\
\hline Arte & $0 \%$ & $0 \%$ & $2 \%$ & $2 \%$ & $0 \%$ & $1 \%$ & $1 \%$ & $0 \%$ & $0 \%$ & $0 \%$ & $0 \%$ & $0 \%$ & $0 \%$ \\
\hline $\begin{array}{l}\text { Marketing, Economía, } \\
\text { Administración y Negocios }\end{array}$ & $14 \%$ & $20 \%$ & $13 \%$ & $8 \%$ & $18 \%$ & $6 \%$ & $13 \%$ & $41 \%$ & $46 \%$ & $39 \%$ & $30 \%$ & $6 \%$ & $32 \%$ \\
\hline TIC & $1 \%$ & $0 \%$ & $0 \%$ & $4 \%$ & $4 \%$ & $0 \%$ & $2 \%$ & $2 \%$ & $4 \%$ & $1 \%$ & $6 \%$ & $2 \%$ & $3 \%$ \\
\hline Otros & $0 \%$ & $2 \%$ & $1 \%$ & $2 \%$ & $0 \%$ & $6 \%$ & $2 \%$ & $10 \%$ & $5 \%$ & $1 \%$ & $18 \%$ & $0 \%$ & $7 \%$ \\
\hline $\begin{array}{l}-1-1-1- \\
\text { Totales }\end{array}$ & $100 \%$ & $100 \%$ & $100 \%$ & $100 \%$ & $100 \% *$ & $100 \%$ & $100 \%$ & $100 \%$ & $100 \%$ & $100 \%$ & $100 \% *$ & $100 \%$ & $100 \%$ \\
\hline
\end{tabular}

Fuente: Elaboración propia 2011.

La Commission on Public Relations Education (CPRE, 2006), en el informe The Professional Bond, señala que la enseñanza ideal de las Relaciones Públicas debiera incluir un mínimo de cinco cursos, entre ellos Teoría y surgimiento de las RR. PP.; Investigación, medición y evaluación de las RR. PP.; Escritura y producción de RR. PP.; Experiencia de trabajo supervisado en RR. PP.; y cursos en Derecho y Ética, planeación y gestión, casos de estudios o campañas. Estas recomendaciones, en su mayoría, están presentes en los planes de estudio de las universidades españolas. Las ausentes son Investigación en RR. PP.; Derecho y Ética en los currículos de la Universidad Autónoma de Barcelona y de la Universidad de Navarra.

Del $80 \%$ de las disciplinas que forman parte de la didáctica de las RR. PP. sobresalen (tabla 1): Comunicación (26\%), Publicidad (22\%), Mercadotecnia, Economía, Administración y 
Negocios (13\%).

Aunque la CPRE (2006) y el IPRA (1990) establecieron incluir otras disciplinas como las artes liberales, letras y ciencias, estas tienen escasa presencia. Las Ciencias Sociales alcanzan un 10\%, mientras que Filosofía y Letras sólo un 2\%. Las Tecnologías de Información y Comunicación (TIC) quedan reducidas a un $2 \%$. Aún se observa la ausencia de asignaturas como Contabilidad, Finanzas, Negocio internacional y Estudios étnicos y feministas, tal como se refleja en el estudio de la enseñanza de las Relaciones Públicas por Xifra (2007).

\subsection{El currículo mexicano}

Las asignaturas de Relaciones Públicas tienen una menor presencia en los planes de estudio que en España. Los resultados revelan que un 14\% de asignaturas son de RR. PP., mientras que las interdisciplinarias alcanzan un $86 \%$. De estas disciplinas, un $32 \%$ corresponden a Mercadotecnia, Economía, Administración y Negocios; un 23\% a Comunicación; y un $14.8 \%$ a Ciencias Sociales y Humanidades.

Tal como ocurre en España, no figuran en el currículo mexicano las siguientes asignaturas: Investigación, medición y evaluación de las RR. PP.; Derecho, Ética y Artes; y los Estudios étnicos y feministas. Pero sí lo hacen Contabilidad y Finanzas.

Existen varios factores que pueden ayudar a determinar la tendencia en la enseñanza de las Relaciones Públicas en España y México, desde su propia naturaleza interdisciplinaria, la propia filosofía de la universidad (IPRA, 1990), las necesidades del entorno económico, político y social en donde se ofertan, la facultad a la que pertenecen y los intereses propios de los gestores académicos, entre otros.

Las RR. PP. en España pertenecen al área de las Ciencias Sociales y Jurídicas, mientras que en México, a las Ciencias Sociales y Administrativas. Se enseñan en diferentes facultades y centros. El IPRA (1990) no recomienda un lugar específico para la disciplina, empero, sugiere que las Relaciones Públicas sean enseñadas como una ciencia social aplicada con énfasis académico y profesional.

En cuanto a las denominaciones propias de los grados y licenciaturas de nuestra muestra, las RR. PP. aparecen en segundo término: le anteceden la Comunicación, Publicidad y Mercadotecnia. (Apéndice A).

Como ya se comentó anteriormente, las disciplinas no relacionadas directamente con las Relaciones Públicas tienen una mayor presencia en las universidades mexicanas ( $86 \%$ ) que en las españolas $(80 \%)$.

En España, destaca la Universidad de Navarra con un 93\% de asignaturas interdisciplinares. La Comunicación adquiere mayor relevancia con un 53\% y la Mercadotecnia, Economía, Administración y Negocios con un 18\%. Esta presencia es un indicador significativo que determina la orientación del grado de Publicidad y Relaciones Públicas hacía la Comunicación y la Mercadotecnia, ya que (en el caso de la enseñanza de las RR. PP.) 
apenas figura en el currículo.

En el caso de la Universidad de Barcelona, predominan las asignaturas de Mercadotecnia, Economía, Administración y Negocios con un 20\% y en la Universidad de Navarra con un 18\%. En la Autónoma de Barcelona y la Complutense de Madrid se enfatiza la Publicidad con un 34\% y 33\% respectivamente. En la Universidad Jaume I, la Comunicación figura con un 37\% y en la Universidad Ramón Llull con un 31\%.

En las universidades mexicanas se evidencia una orientación hacia la Mercadotecnia, Economía, Administración y Negocios en cuatro de las universidades (32\%): Autónoma del Estado de Morelos, Autónoma de Guerrero, del Valle de México y Veracruzana. Este predominio representa poco más del doble si se compara con el caso español (13\%). Solo en la Universidad Latinoamericana se enfoca a la comunicación con un $64 \%$.

A partir de estas cuatro variables (la rama de conocimiento, el centro de adscripción, la denominación de los estudios y el currículo diseñado), se puede afirmar que la enseñanza de las Relaciones Públicas en España se complementa con Comunicación (26\%), Publicidad (22\%), y Mercadotecnia, Economía, Administración y Negocios (13\%). En lo que respecta a México, las RR. PP. se complementan con Mercadotecnia, Economía, Administración y Negocios (32\%) Comunicación (23\%) y Ciencias Sociales (14.8\%). La Publicidad apenas tiene presencia con un $4.8 \%$.

\section{Conclusiones}

Este estudio exploratorio ha comparado la enseñanza universitaria de las Relaciones Públicas en España y México, analizando un total de 11 universidades: 6 de España y 5 de México. Las conclusiones pueden resumirse de la siguiente manera:

- En España, los estudios de comunicación son homogéneos y forman parte de la rama de las Ciencias Sociales y Jurídicas, mientras que en México predominan las Ciencias de la Comunicación y carreras afines independientes dentro las Ciencias Sociales y Administrativas.

- En España, las Relaciones Públicas están adscritas a las Facultades de Información y Comunicación. En México ninguna depende de una Facultad de Comunicación.

- Los estudios de comunicación en la Península son verificados y evaluados por una sola instancia, la Agencia Nacional de Evaluación y Calidad (ANECA), mientras que en México existen tres organismos acreditadores.

- El número de créditos en España ha sido unificado con el Plan Bolonia a 240. En México no existe un sistema común de asignación de créditos para los estudios. La media de créditos de nuestro objeto de estudio es de 363. La recomendada es entre 300 y 450.

- Con el Espacio Europeo de Educación Superior, las asignaturas de Relaciones Públicas han aumentado su presencia (20\%), a solo seis puntos de Comunicación (26\%) y a dos 
puntos de Publicidad (22\%). Estos datos difieren de estudios anteriores realizados con asignaturas de licenciatura (Xifra, 2007) o asignaturas de licenciatura y grado (Matilla, Hernández \& Losada, 2010), que situaban a las RR. PP. en peor situación.

- En México, la presencia de asignaturas en Relaciones Públicas alcanza el 14\%, por detrás de Mercadotecnia, Economía, Administración y Negocios (32\%), Comunicación (23\%) y Ciencias Sociales y Humanidades (14.8\%). La Publicidad apenas alcanza un 5\%.

- Solo en una universidad española se aborda la multiculturalidad (Sriramesh y Hornaman, 2006) y la comunicación para el desarrollo. En ninguna la migración, los estudios étnicos y feministas (Xifra, 2007) y el community manager. En su mayoría, las universidades españolas y mexicanas consideran algunos de los cursos mínimos que recomienda la Commission on Public Relations Education. La Universidad de Barcelona y la Universidad Ramón Llull los incluyen en su totalidad.

- El IPRA (1990) recomienda que las Relaciones Públicas sean enseñadas como una aplicación de las Ciencias Sociales con un énfasis académico y profesional. Sin embargo, las universidades españolas y mexicanas reflejan su limitada presencia: $10 \%$ y $14.8 \%$ respectivamente.

- En ambos países, la educación para el desarrollo humano está en desventaja.

\section{Limitaciones y líneas futuras}

El limitado número de planes de estudio analizados supone una representatividad mínima para determinar los modelos educativos de las Relaciones Públicas. En el caso mexicano, se añade que no se contó con el número de créditos distribuidos para cada asignatura en dos universidades: la Latinoamericana y la Autónoma del Estado de Morelos, esta última está reformando su plan de estudios.

Como líneas futuras, se propone la ampliación de la muestra al resto de universidades españolas (32) y mexicanas (20) que ofertan carreras de Relaciones Públicas. En una segunda etapa, se podría analizar el ámbito europeo, norteamericano y el resto de América Latina. Finalmente, una vez implantado el nuevo plan de estudios de Publicidad y Relaciones Públicas en todas las universidades españolas, se podrá contar con los descriptores de las asignaturas para profundizar en los contenidos, metodología utilizada, evaluación y bibliografía. En definitiva, una delimitación del campo de conocimiento de las Relaciones Públicas nos ayudará a avanzar en la investigación y sobre todo legitimar su presencia en el sistema educativo universitario y en el ámbito profesional. 
Apéndice A: Facultad y centros de adscripción de las universidades españolas y mexicanas.

\begin{tabular}{|c|c|c|}
\hline INSTITUCIÓN & FACULTAD / CENTRO & GRADO / LICENCIATURA \\
\hline Universidad Autónoma de Barcelona & Ciencias de la Comunicación & Publicidad y Relaciones Públicas \\
\hline Universidad de Barcelona & Escuela Superior de Relaciones Públicas & Publicidad y Relaciones Públicas \\
\hline Universidad Complutense de Madrid & Ciencias de la Información & Publicidad y Relaciones Públicas \\
\hline Universidad de Navarra & Comunicación & Publicidad y Relaciones Públicas \\
\hline Universidad Ramón Llull & Ciencias de la Comunicación & Publicidad y Relaciones Públicas \\
\hline Universidad Jaume I & Ciencias Humanas y Sociales & Publicidad y Relaciones Públicas \\
\hline Universidad Autónoma de Guerrero & $\begin{array}{l}\text { Unidad Académica de Ciencias } \\
\text { de la Comunicación }\end{array}$ & Mercadotecnia y Relaciones Públicas \\
\hline $\begin{array}{l}\text { Universidad Autónoma del } \\
\text { Estado de Morelos }\end{array}$ & Ciencias Sociales y Administrativas & Relaciones Públicas \\
\hline Universidad del Valle de México & División de Ciencias Sociales & Relaciones Públicas \\
\hline Universidad Latinoamericana & $\begin{array}{l}\text { Escuela de Comunicación } \\
\text { y Relaciones Públicas }\end{array}$ & Comunicación y Relaciones Públicas \\
\hline
\end{tabular}

Fuente: Elaboración propia.

Apéndice B: Puntos abordados por asignaturas y disciplinas en instituciones españolas.

REL A C I O N E S PÚBLICAS
Aplicación de las RR. PP.; Comunicación de crisis; Comunicación e imagen en las organizaciones; Diseño, creatividad, economía y estadísticas de las RR. PP.; Estrategias y actualidad en RR. PP.; Formación de portavoces; Gestión de cuentas; Inglés especializado en RR. PP.; Métodos de investigación aplicada a las RRPP; Protocolo y gestión de eventos; Relaciones con los medios; Reputación corporativa y responsabilidad social de las empresas; Psicología de la percepción aplicada a las RR. PP. y Teoría y origen de las RR. PP.

\section{COMUNICACIÓN}

Comunicación de moda y cultura; Comunicación electoral; Comunicación integrada de marcas y cultura visual; Comunicación intercultural; Comunicación internacional; Comunicación financiera; Comunicación global; Comunicación oral y escrita; Comunicación política; Comunicación para el desarrollo; Comunicación visual en el periodismo; Comunicación y tendencias; Derecho de la comunicación; Dirección de actores; Diseño periodístico avanzado; Estrategias de comunicación y moda; Estructura y mercados de la comunicación; Estética en medios audiovisuales y publicidad; Ética de la comunicación; Fundamentos de comunicación estratégica y gestión de proyectos; Historia y teoría de la comunicación; Inglés especializado en cine y televisión; Introducción al cine y a la televisión; Lengua española y discurso; Lenguajes comunicativos y audiovisuales; Métodos de investigación en comunicación; Narrativa audiovisual; Propaganda; Psicología; Sociología de la comunicación; Teoría de la imagen; Teoría y técnica de la fotografía. 
PUBLICIDAD
Creatividad y diseño en publicidad; Deontología y derecho publicitario; Economía y estadística aplicada a la publicidad; Inglés especializado en publicidad; Lengua española en la publicidad; Métodos de investigación y gestión documental en publicidad; Marketing aplicado a la publicidad; Planeación estratégica; Realización audiovisual publicitaria; Redacción publicitaria (narrativa publicitaria); Psicología de la percepción aplicada a la publicidad; Sistemas publicitarios contemporáneos y Teoría, origen y estructura de la publicidad.
PERIODISMO

Periodismo religioso, Redacción periodística y Teoría del periodismo.
CIENCIAS SOCIALES Y HUMANIDADES
Antropología; Historia del mundo actual; Métodos y técnicas de investigación social; Sistema político contemporáneo; Relaciones internacionales; Humanística y Derecho.

FILOSOFÍA Y LETRAS

Deontología profesional; Ética de las organizaciones; Filosofía del pensamiento y Literatura.

ARTE Arte contemporáneo, Teoría e historia del arte contemporáneo.

MARKETING,

ECONOMÍA,

ADMINISTRACIÓN

Y NEGOCIOS
Comportamiento del consumidor; Cultura de las organizaciones; Dirección estratégica y liderazgo; Gestión económica y financiera; Gestión de marketing; Marketing social y político y Recursos humanos.

TIC

Comunicación multimedia; Comunidades virtuales, Internet para creativos; Redes sociales y TIC.

Fuente: Elaboración propia.

Apéndice C: Puntos abordados por asignaturas y disciplinas en instituciones mexicanas.

RELACIONES PÚBLICAS
Administración de las Relaciones Públicas; Aplicación de las Relaciones Públicas; Campañas de difusión y organización de eventos; Comunicación organizacional e imagen pública; Relaciones públicas internacionales y especializadas; y Teoría y orígenes de las Relaciones Públicas.
Análisis y evaluación de medios; Cabildeo y propaganda; Comunicación audiovisual; Comunicación gráfica; Comunicación oral y escrita; Elementos de lingüística y semiótica; Estudios de opinión pública; Fundamentos de economía aplicados a la comunicación; Gestión de la comunicación y medios de comunicación; Orígenes y teoría de la comunicación; Planeación de campañas; Psicología de la comunicación; Sociología de la comunicación; Técnicas de expresión gráfica y Teoría de la propaganda. 
PUBLICIDAD Creatividad, Empresa publicitaria; Fundamentos de publicidad; Habilidades del pensamiento crítico y creativo; Publicidad y propaganda; y Semiótica publicitaria.

PERIODISMO Géneros periodísticos informativos, interpretativos y de opinión.

$\begin{array}{ll}\text { CIENCIAS SOCIALES } & \text { Entorno sociopolítico y económico de México; Fundamentos antropológicos y } \\ \text { Y HUMANIDADES } & \text { sociológicos; Metodología de la investigación; Principios generales del derecho } \\ \text { (mercantil, trabajo, fiscal); Sicología social; Sociedad y desarrollo de México y el } & \text { mundo; Teoría social y valores humanos. }\end{array}$

MARKETING,

ECONOMÍA,

ADMINISTRACIÓN

Y NEGOCIOS
Comercio internacional; Contabilidad y finanzas; Derecho económico; Desarrollo organizacional; Emprendurismo, dinámica grupal y teoría del conflicto; Fundamentos de administración y gestión organizacional; Gestión de calidad en el servicio; Gestión del marketing integral; Gestión de negocios; Investigación de mercados; Marco jurídico de la empresa; Mercadotecnia internacional; Organización de restaurantes y bares; Planeación estratégica prospectiva; Principios económicos y estadística; Relaciones obrero patronal; Sistemas de calidad; Sistema político nacional e internacional; Tendencias demográficas y neoeconómicas; Teorías del Estado, administrativas, económicas; Desarrollo, globalización y empresa.

TIC

Introducción a la computación y tópicos de actualidad; Sistemas de telecomunicación e informática.

Fuente: Elaboración propia.

\section{Fuentes consultadas}

Arceo, A. (2004). "Influencias histórico-profesionales en el concepto de Relaciones Públicas”, pp. 57-78. En Arceo, J. (ed.). Las Relaciones Públicas en España. Madrid: McGraw Hill.

Bonilla, C. (2004). Relaciones Públicas, factor de competitividad para empresas e instituciones. México D. F.: Continental.

Castillo, A y Xifra, J. (2006). "Investigación bibliométrica de las tesis doctorales sobre Relaciones Públicas (1965-2005)”. Anàlisi, quaderns de comunicación i cultural, 34, 141-161.

"Catálogo de carreras de licenciatura en Universidades y Tecnológicos" (2007). Extraída el 3/ IX/2011. Asociación Nacional de Universidades e Instituciones de Educación Superior: www.anuies. $\mathrm{mx}$

Commission on Public Relations Education (2006). The Professional Bond. Estados Unidos. Extraída el 3/IX/2011 desde www.commpred.org

Flores, M.; Roca, D. y Tena, D. (2010). “La congruencia profesional de las competencias específicas 
del grado en Publicidad y Relaciones Públicas de las universidades públicas de Cataluña”, pp. 5465. En Cabezuelo, F. y Sierra, J. (coords.). Competencias y perfiles profesionales en los estudios de Ciencias de la Comunicación. Madrid: Fragua.

Fuentes, R. (2010). "Maestría en Comunicación en México: análisis comparativo de sus perfiles de producción y reproducción académica". Comunicación y Sociedad, 14, 13-48.

Grunig, J. y Hunt, T. (2003). Dirección de Relaciones Públicas. Barcelona: Gestión 2000.

IPRA (1990). "Public Relations Education: recommendations and standards". Gold Paper, 7. Londres.

L’Etang, J. (2009). Relaciones Públicas, conceptos, práctica y crítica. Madrid: UOCpress.

"Libro Blanco sobre títulos de grado en comunicación” (2005). Extraída el 3/IX/2011. Agencia Nacional de Evaluación de la Calidad y Acreditación: www.aneca.es

Long, R. (2004). “The other 'New' Mexico Public Relations Accelerates the Move to a Legitimate Democracy", pp. 43-62. En Tilson, D. y Alozie, E. (eds.). Toward the Common Good: Perspectives International Public Relations. Estados Unidos: Pearson Education.

Magallón, S. (2004). "La educación universitaria de las Relaciones Públicas en España”, pp. 375380. En Arceo, J. (coord.). Las relaciones públicas en España. Madrid: McGraw Hill.

Marques, G. (2009). "Public Relations in Portugal. An analysis of the profession through the undergraduate curriculum”. Public Relations Review, 35, 328-330.

Matilla, K; Hernández, S. y Losada, J. (2010). "La oferta formativa de Relaciones Públicas en los nuevos grados universitarios en España (2009-10)". Red Académica Iberoamericana de Comunicación, 7, 124-159.

Molleda, J. (2001). "International Paradigms: the Latin American School of Public Relations". Journalism Studies, 2(4) 513-530.

Noguero, A. (2004). "Principales acontecimientos en la evolución de las Relaciones Públicas en España”, pp. 337-357. En Arceo, J. (coord.). Las relaciones públicas en España. Madrid: McGraw Hill.

Olmedo, S.; Álvarez, A. y Ruiz, I. (2010). "Estudios de comunicación en el Espacio Europeo de Educación Superior (EEES). Nuevos contenidos, nuevos perfiles profesionales”, pp. 66-81. En Sierra, J. (coord.). Los estudios de Ciencias de la Comunicación en el EEES. Madrid: Fragua.

Pratt, C. y Ogbondah C. (1996). "International Public Relations Education: U.S. Issues and Perspective", pp. 381-395. En Culbertson, H y Chen, N. (eds.). International Public Relations, a comparative analysis. New Jersey: Lawrence Erlbaum Associates.

Sriramesh, K., y Hornaman, L. B. (2006). "Public Relations as a profession, an análisis of curricular content in the United States". Journal of Creative Communications, 1:2.

“¿Qué es la ACCECISO?” (2006). Extraída el 3/IX/2011. Asociación para la Acreditación y Certificación en Ciencias Sociales: http://www.acceciso.org.mx/esp/index.php

Wright, D. (1991). Current reflections about Public Relations Education in the United States. Presentado en el International Education Meeting. Toronto, Canadá.

Xifra, J. (2007). “Undergraduate public relations education in Spain: Endangered species?”. Public Relations Review, 33, 206-213. 